Tangling with hypothermia

Jesús Avila and Javier Díaz-Nido

Hypothermia resulting from impaired glucose metabolism may be one of the factors that contribute to hyperphosphorylation of the protein tau and subsequent neuronal dysfunction.

Aging is a major risk factor for the development of Alzheimer disease, the most prevalent cause of dementia in the elderly. With age, hyperphosphorylated tau accumulates and aggregates into neurofibrillary tangles in the brains of people with $A D$ and other neurological disorders referred to as tauopathies. Whereas normal tau stabilizes the neuronal cytoskeleton, hyperphosphorylated tau may disrupt microtubules and interfere with intraneuronal organelle transport, ultimately leading to dysfunction of synapses, degeneration of neurons and cognitive impairment. There is therefore great interest in unraveling the mechanisms that control tau phosphorylation.

In a study in the March 10 issue of the Journal of Neuroscience, Planel and colleagues examine the relationship between disturbed glucose metabolism and tau hyperphosphorylation. It is well known that affected areas in the brain of patients with Alzheimer disease show significant reductions in glucose and energy metabolism, which seem to correlate spatially with the accumulation of hyperphosphorylated tau. Planel and colleagues had previously reported that starvation, which lowers glucose utilization, induced a reversible hyperphosphorylation of tau in the hippocampus and cortex of mice. The authors now extend these observations by showing that treatments that reduce glucose metabolism also result in hypothermia. Crucially, they traced the increase in tau phosphorylation to an inhibitory effect of temperature, not of glucose metabolism per se, on protein phosphatase activity.

Several protein kinases, including glycogen synthase kinase-3 (GSK-3), cdk5 and Jun kinase phosphorylate tau, whereas its dephosphorylation is mainly catalyzed by protein phosphatase 2A (PP2A). The authors found that the activity of phosphatases, including PP2A, decreased exponentially at low temperatures in an in vitro system, whereas the activity of protein kinases diminished only linearly. Hypothermia, therefore, resulted in a predominance of kinase over phosphatase activity.

By contrast, altering glucose concentrations at constant temperature did not have any effect on tau phosphorylation. These findings are in line with studies showing that starvation in mice leads to hyperphosphorylation of tau through a marked inhibition of phosphatases. Other studies have also found that tau hyperphosphorylation occurs during hibernation and cold stress in some animals.

What could be the relevance of these findings to Alzheimer disease? Although the effect of temperature needs to be confirmed in vivo, and the mechanism whereby the reversible hyperphosphorylation of tau can ultimately lead to the formation of tangles remains to be established, there are intriguing parallels between the mouse model and the human disease.

In the mouse, starvation and hypothermia trigger a reversible hyperphosphorylation of tau that is reminiscent of presymptomatic stages of Alzheimer disease. In both cases, for instance, hyperphosphorylated tau localizes to axonal tracts in the hippocampus and cortex, but not to neuronal cell bodies or dendrites.

But in the brains of patients with Alzheimer disease, the deficit in PP2A may be more pronounced, as both the expression level and the activity of PP2A are diminished. 
Moreover, in mice where PP2A is directly inhibited in vivo by pharmacological or genetic means, a fraction of hyperphosphorylated tau accumulates as perinuclear aggregates that resemble 'pre-tangles'.

Elderly people tend to regulate body temperature less efficiently than young people. Moreover, hypothermia could be an issue with diabetic patients-another population at risk for Alzheimer disease-who exhibit impaired thermoregulation and may also experience episodes of hypothermia after insulin injections. In this case, however, we would be naive to conclude that diabetes enhances the risk of Alzheimer disease exclusively through hypothermia.

Indeed, there is ample evidence linking other aspects of insulin signaling with the modulation of tau phosphorylation in neurons. For example, mice lacking the insulin receptor in neurons have increased GSK3 activity, normal levels of PP2A expression and significant tau hyperphosphorylation. These results suggest that neuronal insulin resistance may also facilitate tau hyperphosphorylation through activation of kinases without any alteration in phosphatase activity-a pattern opposite to that identified by Planel et al. Whether this reverse pattern also occurs in patients with diabetes remains to be investigated.

Other studies also point to GSK-3 as responsible for tau hyperphosphorylation. Transgenic mice overexpressing this kinase have hyperphosphorylated tau and impaired cognitive abilities. Rats treated with phosphatidylinositol-3 kinase and protein kinase $C$ inhibitors, which block signal transduction in response to insulin, IGF-I and other trophic factors, have increased GSK-3 activity, hyperphosphorylated tau and impaired spatial memory.

On the basis of all of this evidence, we should not rush to argue for a causal role of hypothermia-induced tau hyperphosphorylation in Alzheimer disease. Instead, we might think about hypothermia as another risk factor for the disease. In addition, these studies point to a complex interplay of elements that modulate the phosphorylation level of tau (Fig. 1) through the inhibition of PP2A and activation of GSK-3 and other kinases in response to a variety of age-related neuroendocrine alterations.

Accordingly, the inhibition of protein kinases that phosphorylate tau or the activation of PP2A may be promising approaches for therapeutic intervention in Alzheimer disease and other tauopathies. At the moment, some progress is being made to develop specific kinase inhibitors aimed at curbing tau hyperphosphorylation. The provocative study by Planel and colleagues has situated hypothermia as an additional factor in this scheme. 


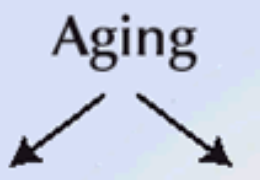

IGF-1 $\downarrow \quad$ Type II diabetes

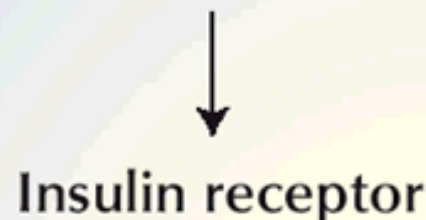
desensitization

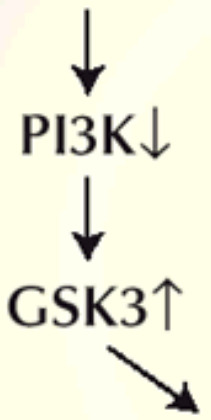

Starvation

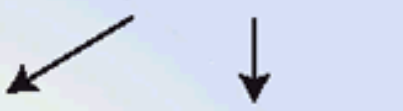

Insulin $\downarrow$ Reduced glucose metabolism

Hypothermia

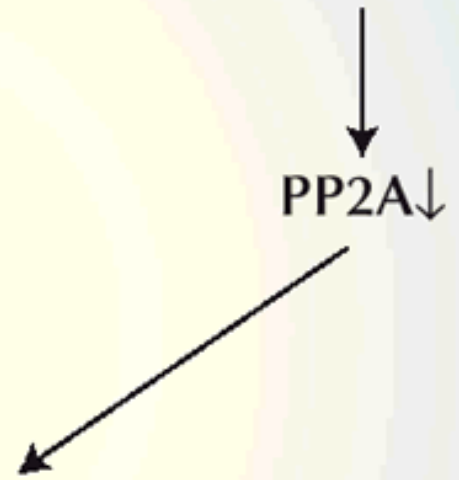

2

Tau hyperphosphorylation

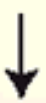

\section{Synaptic dysfunction}

Neurodegeneration

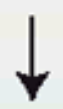

Dementia

Figure 1 Two different routes to tau phosphorylation, a modification that can result in dementia.

Jesús Avila and Javier Díaz-Nido are at the Centro de Biología Molecular "Severo Ochoa" (CSICUAM), Universidad Autónoma de Madrid, 28049 Madrid, Spain. 